\title{
Congenital Head \& Neck Anomalies A Clinical and Anatomical Study
}

\author{
T. Sobha Devi ${ }^{1}$, P. Hari Krishna ${ }^{2}$ Sabeeha naaz ${ }^{3}$ \\ ${ }^{1}$ Assistant professor, Dept of Anatomy, S.V. Medical College, Tirupati NTRUHS India. \\ ${ }^{2}$ Assistant professor, Dept of E.N.T, S.V.M.C \& S.U.R.R.G.H, Tirupati NTRUHS India. \\ ${ }^{3}$ Final year M.B.B.S, Gandhi Medical College, secunderabad,NTRUHS,India.
}

\begin{abstract}
In this article we have presented three cases of congenital head \& neck anomalies and discussed the possible embryological etiology. These cases include a unilateral right sided isolated anotia in a male baby aged 4 months nasal dermoid sinus cyst in an adult female, adolescent female with high arched palate, stenosis of nares, presenting with stridor. Study involved cases presenting with congenital anomalies surviving into adulthood with attendant implications over the quality of life of these subjects.
\end{abstract}

Key words: Isolated anotia, High arched palate, Laryngeal web, Nasal dermoid sinus cyst, Congenital head and neck anomalies.

\section{Introduction}

Head and neck congenital anomalies are rare. Cases surviving into adulthood were also uncommon. Quality of life of individuals surviving with anomalies is interesting to the surgeon and also scientific community. It involves reconstruction and rehabilitation perspective which are challenging procedures with regard to their outcome. Study focus on the embryological aspects of the anomalies.

\section{Materials and methods}

Study was undertaken in the S.V. Medical college and SVRRGGH Tirupati, A.P between the years 2010-2013 in the Dept of E.N.T. patients were screened for congenital head and neck anomalies using routine O.P procedures and recording the clinical history.

\section{Observations}

Case 1: 4 months year old male baby was bought to the E.N.T O.P(Figures 1,2) by the parents with complaints of congenital absence of right external ear. Left ear is normal with well formed auricle and external auditory meatus, tympanic membrane. Not associated with any other congenital anomalies. No history of antenatal infections to mother, received immunisation during pregnancy. No history of consanguinity. Belongs to low socio economic group. Maternal illiteracy. No family history of the anomaly previously. Baby was second child to the mother, first child was normal.

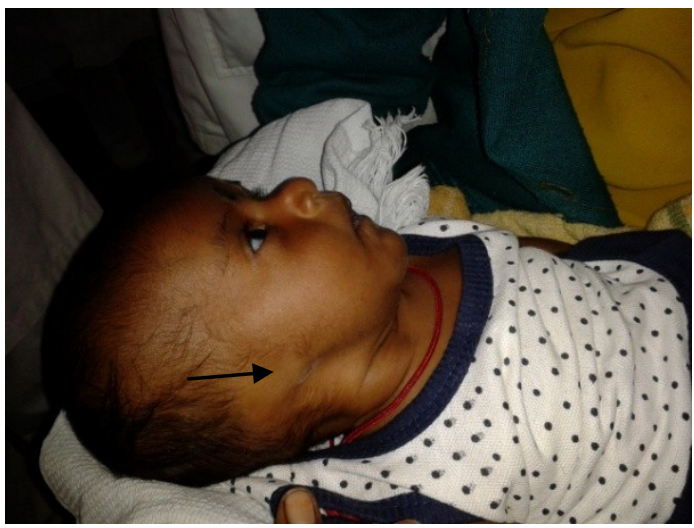

Figure 1 showing Rt. Sided Anotia

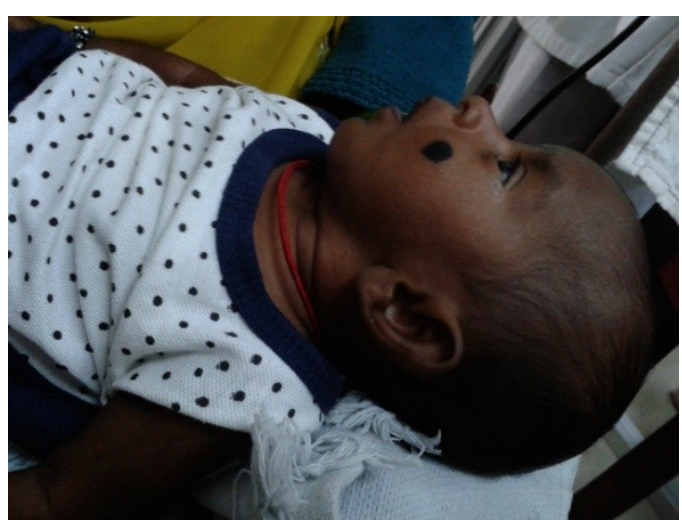

Figure 2 showing Lt. Sided ear is normal

Case 2: Female patient aged 25 years(figure 3) came with complaints of discharging from an opening between the medial canthus of right eye $\&$ bridge of nose. On examination scarring with sinus seen. No active discharge. Diagnosed as Nasal dermoid sinus cyst. No intracranial extension, no other associated anomalies 


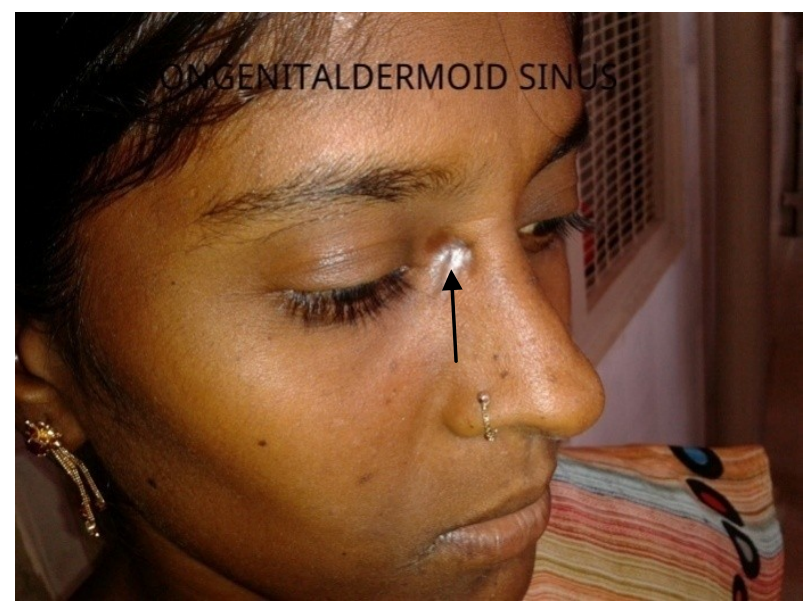

Figure 3 Arrow shows Nasal dermoid sinus cyst

Case 3: Female patient aged 21 years 9(fig. 4, 5) came to ENT O.P with the complaint of stridor. Direct laryngoscopy revealed posterior glottis web. Associated anomalies found are high arched palate, stenosis of right sided nares, history of consanguinity present.

Patient was admitted for microlaryngeal surgery under general anaesthesia. Larynx approached by anterior laryngo fissure, larynx opened, posterior glottis web resected, silastic keel kept anteriorly to prevent adhesions. Larynx closed in layers. Keel removed after 2 weeks.postoperatively uneventful, no infections,stridor resolved.

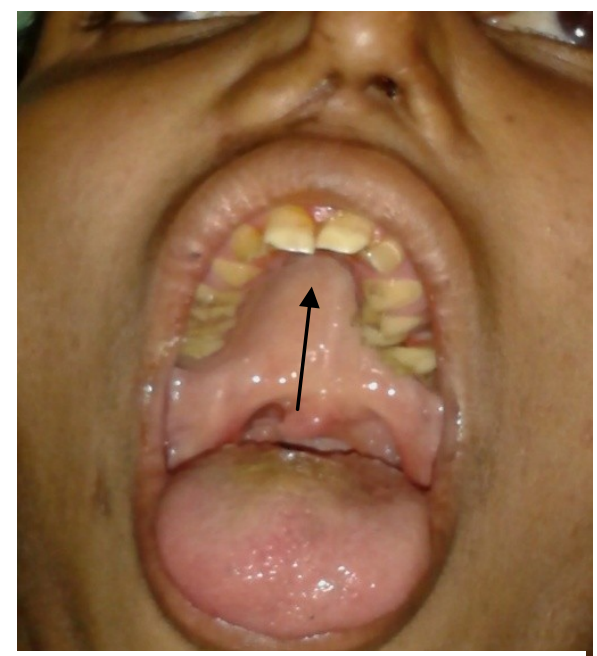

Figure 4 Arrow shows high arched palate

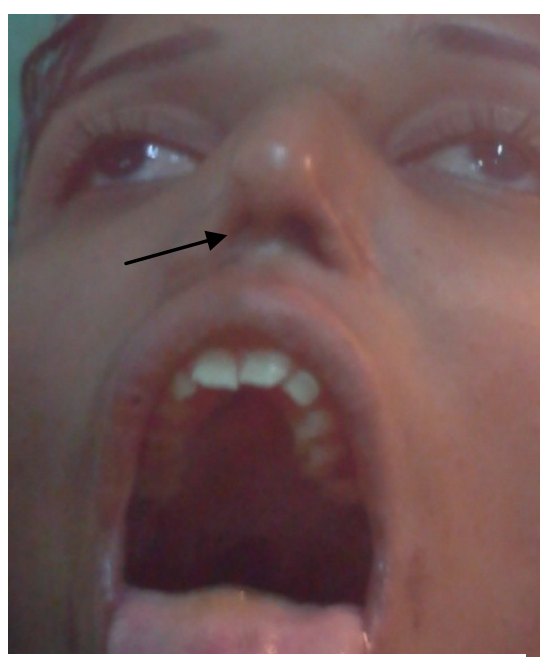

Figure 5 Arrow shows Rt.

Sided Nasal stenosis

\section{Discussion}

External ear develop from 6 mesodermal hillocks, 3 each contributed by mandibular, hyoid arches. They fuse to form definitive auricle. As fusion of auricular hillocks is complicated, developmental abnormalities of auricle are complicated, developmental anomalies of auricle are common. Initially external ears are in the lower neck region, with the development of mandible they ascend to the side of head at the level of eyes. External ear defects are common. They are significant from stand standpoint of the psychological and emotional trauma. All of the frequently occurring chromosomal syndromes have ear anomalies as one of their character.

Anotia (type IV microtia-anotia) according to J. Harris et al(Ref. 9) et al in unilateral isolated cases right sided is prevalent then left sided one. According to above study male prevalence is common in isolated cases which supports the present case. Paput L et al (Ref. 11)studied on the possible multifactorial threshold model in the origin of isolated microtia/anotia and came to the hypothesis that gene-environmental interaction may be the explanation for congenital abnormality group According to Shaw GM et al(Ref 16) the prevalence of 
anotia/microtia was 2.50 per 10,000 live births and stillbirths. Isolated cases, i.e., those live births and stillbirths with no other accompanying anomaly diagnoses, revealed a prevalence of 0.63 per 10,000 , whereas among non isolated cases, the prevalence was 1.53 per 10,000 live births and stillbirths. Prevalences of isolated anotia/microtia were substantially higher among U.S.-born Hispanics, foreign-born Hispanics, and Asians compared to non-Hispanic whites, with relative risks ranging from three- to seven-fold. Similar prevalences for maternal race/ethnicity (except for Asians) were observed for live births and stillbirths with nonisolated cases of anotia/microtia, but the magnitude of risks were lower than those observed for isolated cases. Prevalence of non isolated anotia/microtia was substantially lower among women whose education was 12 years or more.

P Mastroiacovo et a(Ref 10)l studied The epidemiology and genetics of microtia-anotia (M-A) using data collected from the Italian Multicentre Birth Defects Registry (IPIMC) from 1983 to 1992 found that mothers with parity 1 had a higher risk of giving birth to an MMI(Multiformed infants) with M-A, and that mothers with chronic maternal insulin dependent diabetes were at significantly higher risk for having a child with M-A. MMI with M-A had higher rates of prematurity, low birth weight, reduced intrauterine growth, and neonatal mortality than infants with isolated M-A and controls. Babies with isolated M-A had, on average, a lower birth weight than controls; the difference was higher for females. The analysis of pedigrees and familial cases suggests an autosomal dominant trait with variable expression and incomplete penetrance in a proportion of cases, or a multifactorial aetiology. Three cases had consanguineous parents, but the absence of M-A among previous sibs does not support autosomal recessive inheritance. No history of consanguinity in the present case .

Congenital malformations of the external ear are relatively rare, however gradual increase in their frequency has been observed in the last years.Garstcka E et al(Ref 6) studied on 37 patients age 1 to 30 [mean age 12.4 years] with anotia, microtia or aplasia of external ear canal were part of this study. In 17 examined patients $[46.0 \%$ of all examined persons] microtia could be treated as an isolated malformation, however hypoplasia of other elements formed from branchial arches, including mandibula, cheek or oral cavity, was found in 15 examined patients [40.5\%]. In 11 [29.7\%] patients congenital malformations of the external ear were found together with the congenital defects of distant organs (kidney, heart, muscular and skeletal system), and in two patients [5.4\%] the defects involved even two different distant organs. Defects of the distant organs were found more frequently in patients with bilateral malformations of the external ear than in patients with unilateral ear malformation.According to Canfield MA et al(Ref 2) most anotia/microtia cases were in the unilateral and microtia groups, and $45 \%$ were isolated. Several clinical subgroups exhibited higher prevalence in males and among older mothers. Relative to whites, blacks were at lower risk and Hispanics (especially Mexicoborn mothers) were at higher risk for selected types of anotia/microtia.In present study the patient was male.

According to Luquetti et al(Ref 15) the overall prevalences were: microtia-anotia, 2.06 (confidence interval [CI], 2.02-2.10); microtia, 1.55 (CI, 1.50-1.60); and anotia 0.36 (CI, 0.34-0.38). Higher prevalences were observed for the Americas, Northern Europe and Asia, among Hispanics and Asians, and among active ascertainment and hospital-based surveillance programs. Study contributes to the knowledge of the prevalence of microtia-anotia by providing a critical analysis of the existing data. In addition, it supports the need for a coding system that allows complete phenotype characterization of microtia-anotia, including severity and laterality, as well as for further studies on the variation of its frequency related to race and ethnicity. According to Forrester $\mathrm{MB}$ et al(Ref 4$)$ the rates for only a few structural birth defects were substantially greater than expected in association with anotia and microtia. Anotia and microtia rates varied significantly according to maternal race/ethnicity, infant sex, plurality, birth weight, and gestational age.

Nasal dermoid sinus cyst was an uncommon midline anomaly with an embryological origin quite distinct from dermoids elsewhere on the face and requires especially careful approach for management. They are congenital lesions lined by stratified squamous epithelium with normal dermal appendages including hairs and sebaceous glands.they are thought to arise in early embryonic period during closure of anterior neuropore, during development of frontonasal process or during closure of fonticulis nasofrontalis(a potential defect between developing frontal \& nasal bones). According to Wille Blake et al Both sinus, cyst have communication with intracranial components which is not seen in present case.

According to Holzmann D et al(Ref.7) surgical approaches for nasal dermoid sinus cyst to prevent recurrence is simple excision and mobilization of pit, while the proximal part is resected using a coronal transfrontal approach. Relation of nasal fistula to nasal bone is essential considering osteotomy. According toCharrier JB et al(Ref. 3) Thirty children with nasal midline masses and/or sinus ostia were surgically treated in the pediatric ENT and cervicofacial surgery department of Trousseau's children's hospital (Paris-France) between 1995 and 2002. All the patients underwent radiological evaluation including CT scan (including axial and coronal planes) and/or MRI (in all three planes) in search of intracranial extension. Thirteen of our patients presented with a midline cyst only, ten had nasal pit only, and seven had combined anomalies. Preoperative radiology and surgery showed an intracranial extension in 3 patients, which exhibited contact of the cyst with the dura. Recurrence being expected if any dermal tissues were left in place, "one-time-excision management" was the rule. Most present as extracranial lesions in childhood and are effectively removed via open rhinoplasty 
with no recurrence; however, occasionally, they extend intracranially. According to Post $\mathrm{G}$ et al case report presents a unique (unusual) extension of the NDSC into the frontal sinus requiring an osteoplastic flap to access the frontal sinus floor combined with a local midline nasal incision at the sinus tract origin.

According toHacker DC et al(Ref.8) a 56-year-old man presented with a draining sinus in the right medial canthal area and a nasal pit. Imaging studies suggested a nasal dermoid cyst with intracranial extension. He subsequently underwent a craniofacial removal of the lesion with a satisfactory postoperative course. nasal dermoid cysts can remain dormant until the age of 56 suggests that the incidence of this lesion with intracranial extension may be higher than previously reported. A one-stage combined otolaryngologic/neurosurgical procedure is advocated for those lesions that are extra- and intracranial.

Midline congenital nasal lesions are rare, occurring in one out of every 20,000-40,000 births. Of these midline lesions, nasal dermoids are the most common. Multiplanar, high-resolution thin section magnetic resonance imaging allows for excellent soft tissue detail, particularly when intracranial extension is expected. Open rhinoplasty is favored by many authors for excision of dermoids. Transnasal endoscopic excision of nasal dermoids has been reported but is not recommended for dermoids extending into or beyond the falx cerebri. According to Zapata $\mathrm{S}$ et a(Ref.18)1 Imaging of the midface and brain is essential for accurate diagnosis, assessment for any intracranial extension and appropriate surgical planning. Any surgical approach for removal of nasal dermoid cysts should permit adequate access, allow repair of the skull base and cerebrospinal fluid leak, facilitate nasal reconstruction and result in acceptable cosmesis. The head and neck surgeon should be able to consider various surgical approaches to manage these lesions.

Zerris VA et al(Ref. 19) observed two cases.The first patient, a 33-year-old woman, sought care for chemical meningitis. As a child, she was differentiated from her identical twin sister by a dimple on the tip of her nose. The second patient, a 34-year-old man, sought care for new-onset seizures. Since birth, he had a dimple on the tip of his nose. As a child, he had undergone resection of a nasal cyst. Imaging studies in both patients indicated a midline anterior cranial base mass within the falx and a defect in the crista galli. Both patients underwent biorbitofrontal nasal craniotomy. A bifrontal craniotomy was performed first, then removal of the orbitonasal ridge. The dermoid and involved falx were resected. The sinus tract was followed through the crista galli and resected up to the osteocartilaginous junction in the nose. The remainder of the tract was resected via a small incision through the nares. The dura was closed primarily by mobilizing the dura along the sides of the crista galli. After surgery, both patients still possessed their sense of smell.

According to Ted L TewFik et al (Ref.17) embryogenesis related to laryngeal web is due to incomplete recanaliszation of laryngotracheal tube during $3^{\text {rd }}$ month of gestation, leading to different stages of laryngeal webs. Most common type is anterior at the level of vocal folds, less common type is posterior interarytenoid or subglottic web, extreme rare is complete laryngeal atresia. Stridor is typical feature in posterior interarytenoid web.

commonly used classification system for posterior glottic stenosis divides the occurrence into four types. Type I involves an interarytenoid scar band between the vocal folds that is anterior and separate from the posterior interarytenoid mucosa. Type II stenosis involves scarring of the mucosa or musculature of the posterior interarytenoid area. Types III and IV involve unilateral and bilateral cricoarytenoid joint fixation, respectively. Strobovideolaryngoscopy (SVL), rigid and flexible fiberoptic bronchoscopy, electromyography (EMG), radiologic imaging of the neck, larynx, and trachea as well as pulmonary function tests, including flow volume loops, provide important objective measurements of upper airway obstruction. Gallivan GJ(Ref. 5) studied on the treatment utilized a specific contact-tip neodymium-yttrium aluminum garnet (Nd-YAG) laser delivery system to achieve precise cutting, vaporization, and coagulation simultaneously, returning tactile touch technique to the airway/voice surgeon. Completely successful restoration of voice and airway have been maintained for $21 / 2$ years postoperatively.

Chitose $S$ et al(Ref.1) highly recommend the use of a posterior mucosal flap for the treatment of posterior glottic stenosis. This procedure does not require the use of either a laryngofissure or a laryngeal stent. According to Rovó L et al(Ref. 13) After proper mobilization, endoscopic arytenoid lateropexy can be considered as a minimally invasive function-preserving procedure even for severe PGS. This treatment option provides stable improvements in breathing ability and good voice quality without the need for tracheostomy

\section{Conclusion}

Isolated anotia is a rare anomaly. Reconstruction and rehabilitation is usually undertaken beyond 5 years of age, by the time both functional, anatomical evaluation can be done including BAER, PTA. Hearing gain depends on need for reconstruction of middle ear anomalies, inner ear anomalies. Posterior glottis web presents with stridor and voice outcome postoperatively is expected to be good as anterior commisure is not involved. Nasal dermoid sinus cyst poses difficult problem on the operation field, due to evaluation for intracranial extension and post operative recurrence rate is difficult for evaluation. The study will be continued as to 
evaluate for offending agents like infections, toxins, gene mutations to be implicated and clinical presentation at various age levels so as to plan out appropriate rehabilitation, reconstruction requirements.

\section{References}

[1]. Chitose S, Umeno H, Nakashima TEndoscopic surgical treatment of posterior glottic stenosis. J Laryngol Otol. 2009 May;123 Suppl 31:68-71. doi: 10.1017/S002221510900512X. PMID: 19460208

[2]. Canfield MA, Langlois PH, Nguyen LM, Scheuerle AE Epidemiologic features and clinical subgroups of anotia/microtia in Texas. Birth Defects Res A Clin Mol Teratol. 2009 Nov;85(11):905-13. doi: 10.1002/bdra.20626. PMID: 19760683

[3]. Charrier JB, Delattre J, Denoyelle F, Garabédian EN. [Clinical and embryological approaches to nasal dermoid sinus cysts]. Ann Otolaryngol Chir Cervicofac. 2003 Dec;120(6):315-29PMID14730276

[4]. Forrester MB, Merz RDDescriptive epidemiology of anotia and microtia, Hawaii, 1986-2002. Congenit Anom (Kyoto). 2005 Dec;45(4):119-24PMID: 16359491

[5]. Gallivan GJ. Bilateral vocal fold posterior glottic/subglottic stenotic web resected with contact tip Nd-YAG laser.J Voice. 2002 Sep;16(3):415-21PMID: 12395994

[6]. Garstecka A, Betlejewski S, Skonieczka KMicrotia: isolated defect of hearing organ, or syndrome forming collection of abnormalitiesOtolaryngol Pol. 2008;62(5):639-42. doi: 10.1016/S0030-6657(08)70332-9. PMID: 19004275

[7]. Holzmann D, Huisman TA, Holzmann P, Stoeckli SJ. Surgical approaches for nasal dermal sinus cysts. Rhinology. 2007 Mar;45(1):31-5 PMID;17432066

[8]. Hacker DC, Freeman JL. Intracranial extension of a nasal dermoid sinus cyst in a 56-year-old man. Head Neck. 1994 JulAug;16(4):366-71. PMID: 8056582

[9]. Harris J, Källén B, Robert E. The epidemiology of anotia and microtia. J Med Genet. 1996 Oct;33(10):809-13 PMID:8933331

[10]. P Mastroiacovo, C Corchia, L D Botto, R Lanni, G Zampino, and D Fusco Epidemiology and genetics of microtia-anotia: a registry based study on over one million births. J Med Genet. 1995 June; 32(6): 453-457. PMCID: PMC1050485

[11]. Paput L, Czeizel AE, Bánhidy F. Possible multifactorial etiology of isolated microtia/anotia--a population-based study. Int J Pediatr Otorhinolaryngol. 2012 Mar;76(3):374-8. doi: 10.1016/j.ijporl.2011.12.012. Epub 2012 Jan 9 PMID: 22227122

[12]. Post G, McMains KC, Kountakis SEAdult nasal dermoid sinus cyst. Am J Otolaryngol. 2005 Nov-Dec;26(6):403-5. PMID: 16275411

[13]. Rovó L, Venczel K, Torkos A, Majoros V, Sztanó B, Jóri J Endoscopic arytenoid lateropexy for isolated posterior glottic stenosis. Laryngoscope. 2008 Sep;118(9):1550-5. doi: 10.1097/MLG.0b013e31817c0b32. PMID: 18677281

[14]. Langman's medical embryology, $10^{\text {th }}$ edition 2006 LIPPINCOTT WILLIAMS\& WILKINS

[15]. Luquetti DV, Leoncini E, Mastroiacovo P. Microtia-anotia: a global review of prevalence rates. Birth Defects Res A Clin Mol Teratol. 2011 Sep;91(9):813-22. doi: 10.1002/bdra.20836. Epub 2011 Jun 7. PMID: 21656661

[16]. Shaw GM, Carmichael SL, Kaidarova Z, Harris JA. Epidemiologic characteristics of anotia and microtia in California, 19891997Birth Defects Res A Clin Mol Teratol. 2004 Jul;70(7):47PMID: 15259037

[17]. Ted L Tewfik, Congenital Malformations of the Larynx med scape references

[18]. Zapata S, Kearns DB. Nasal dermoids. Curr Opin Otolaryngol Head Neck Surg. 2006 Dec;14(6):406-11. PMID: 17099348

[19]. Zerris VA, Annino D, Heilman CB. Nasofrontal dermoid sinus cyst: report of two cases. Neurosurgery. 2002 Sep;51(3):811-4; discussion 814PMID: 12188963 